\title{
Prospective Engineering Applications of Dynamic Transfer Processes Possessing the Self-Organized Fractal Interfaces
}

\author{
Aliaksandr Yurievich Alevanau, ${ }^{1}$ Olgerd Pavlovich Kuznechik, ${ }^{2}$ \\ and Oleksandr Ivanovych Vyhoniailo ${ }^{3}$ \\ ${ }^{1}$ KTH, ITM/MSE, Division of Energy and Furnace Technology, Brinellvägen 23, 10044 Stockholm, Sweden \\ ${ }^{2}$ BSU, Observatory, Nezalezhnasty Avenue 4, 220030 Minsk, Belarus \\ ${ }^{3}$ Ukrenergoproduct, Balzack Street 8/31, Kiev 02225, Ukraine \\ Correspondence should be addressed to Aliaksandr Yurievich Alevanau; alevanau@kth.se
}

Received 30 December 2012; Accepted 6 May 2013

Academic Editor: Run-Cang Sun

Copyright (c) 2013 Aliaksandr Yurievich Alevanau et al. This is an open access article distributed under the Creative Commons Attribution License, which permits unrestricted use, distribution, and reproduction in any medium, provided the original work is properly cited.

\begin{abstract}
General formulation of practically applicable fundamental approach for development of engineering applications in systems with dynamically appearing and disappearing fractal structures is proposed. The approach is illustrated on the low-temperature pyrolysis of butane/propane mixture being pumped via the liquid tin and bismuth alloy preserved at the temperature $200 \pm 20^{\circ} \mathrm{C}$ in the $\mathrm{U}$ shaped test glass. Other prospective engineering applications of the approach are proposed on the base of analysis of selected experiments described in literature.
\end{abstract}

\section{Introduction}

It is well known from such classical books as [1] that fractals appear in a big variety of natural phenomena. Important emphasis on analysis involving fractals is put to their selforganized character in such works as $[2,3]$. Despite the fact that initially the self-organized dynamics in open systems were considered without special attention to the fractals [4], we would like to note that such basic theory as scale relativity [5] considers the space as a fractal. In the theory [5] the geodesics of elementary particles, which form substances, are considered as having fractal dimensions, which are changing with the scales. Nottale notes in the chapter 2 of his work [5] that the fractal dimension of paths for particles analyzed by Feynman in the quantum scale equals 2 .

What might be found regarding the geodesics of elementary particles in the bigger scales?

The obvious answer to this question is the note that there should be a possibility for interaction with motion of energy along these geodesics, as its fractal characteristics may coincide with the fractal characteristics of the selforganized dynamic systems in the bigger scales. In such a case the feedback of this interaction may be found on the both macroscale of a dynamic system with the fractals, and in the quantum scale of elementary particles and structures, which are formed by the particles in this scale.

Such a possibility is really close to the engineering applications on both scales. Exploration of this hypothetical possibility on several engineering examples is an objective of this paper.

\section{Approaches to the Systems with Hypothetical Interactions between the Self-Organized Structures on Different Scales}

Recent achievements in the experimental researches of the systems, which produce dynamically created and selforganized fractals, allow consideration of such systems as a basis for prospective development of new energy sources. The basic approach for this is in organizing possibilities for energy transfer along the fractal geodesics, which span along several scales, among which one is considered as transmitting 
energy and the other one as receiving it. If the transmitting scale is the quantum microscale, the structures on this scale may undergo changes, which can be classified as their transmutation or nuclear reactions providing with energy of motion for the self-organized structures in the bigger scale. If the self-organization on this bigger scale involves motion of electric charges, the possibility for direct generation of electricity becomes most prospective possibility regarding the approach for creation of new energy sources. In such a case there is no need for the generation of heat from nuclear reactions, then for electricity generation from the heat using steam turbines, and so forth.

The necessary note regarding this ideal possibility should be in careful separation of two stages of the whole process. The first stage is an intentional creation of the self-organized fractal structures in some construction in an engineering scale. These structures have their main task to enter into an interaction with the motion along the fractal geodesics of elementary particles, which form some fuel substance. This interaction has to have disturbing character on the geodesics resulting in a decay or transmutation of the fuel substance. The second stage of the process is to obtain a feedback of this disturbing action to the bigger scale possessing moving and charged structures, which are applicable for extraction of energy coming from the nuclear transmutation in microscale.

These two interconnected tasks have to be joined in the same construction in the bigger scale using the self-organized fractal processes.

We describe two currently available engineering approaches to accomplish these tasks in the following two subsections.

2.1. Retroactive Force Loop Proposed by Nottale. The first approach is to apply the proposal for hydraulic macroscale experiment described by Nottale and Lehner [6]. This approach has its partial support in our own experiments with the low-temperature pyrolysis of gaseous hydrocarbons to be described in the third section of this paper. It assumes the creation of so-called macroquantum oscillating wave packet in the engineering macroscale, which has to obtain its form being dynamically tuned according to the theory [5] to elucidate the fractal character of space and existence in it of so-called generalized quantum potentials [7] appearing opposite to the potentials driving some transfer processes. Such processes in our engineering system may be the processes of dynamically changing heat and mass transfers, which are created by change of global gradients of corresponding physical potentials in the system. For example, with reference to the heat transfer considered in [7] as diffusion, the diffusion driving potential:

$$
\phi_{\text {diff }}=2 D^{2} \nabla\left[\frac{\Delta \sqrt{P}}{\sqrt{P}}\right] .
$$

Corresponding generalized quantum potential:

$$
\frac{Q}{m}=-2 D^{2} \nabla\left[\frac{\Delta \sqrt{P}}{\sqrt{P}}\right] \text {. }
$$

Here, the constant $D$ is equal to $h / 2 m$ for the quantum scale. It may take other values for the other scales. The $h$ is the Plank constant and $m$ is a mass of the system. The constant $D$ is the heat conductivity for a temperature $P$. Q is the quantum-like force of the generalized quantum potential.

The proposal for physical experiment in [6] includes an example of one-dimensional system with the harmonic oscillations represented by the standard equation $x=a \cos w t$. The oscillations should be iteratively governed by the retroactive force loop to approach the form of macroquantum wave packet with its energy written as:

$$
E_{c}=D w+\frac{1}{2} a^{2} w^{2}
$$

Accordingly to the simulations in [6], once the oscillations approach the form governed by the predicted quantumlike force, the macroquantum wave packet becomes more stable with regard to the simulated external perturbations.

From our point of view, such a situation may also bring some side effects to the oscillations in lower scales. We hypothesize in our work [8] that, due to an existence of the dynamically changing global gradients, the atomic and molecular ensembles on micro- and mesoscales may have a special character of harmonic oscillations with slightly deviated potentials between their interacting parts because of link with the oscillations in bigger scales.

Because of this possibility, a wide class of engineering systems utilizing pyrolysis and gasification, which have possibilities for the self-organized oscillations on the fractal geometries spanned on several scales [9], may be applied for research of this link and corresponding energy transfer between the oscillations in different scales.

Main recommendation for engineering applications regarding this possibility is to provide these systems with an intentional modulation of basic energy transfer processes in reactors to direct their internal self-organization towards appearance of the macroquantum wave packets having connection with the same packets and oscillations in lower scales. Such a connection may bring lower activation energies for the reactions of thermochemical decomposition in these reactors.

Obvious note regarding totally different time scales of the presumably connected oscillations in the different spatial scales brings us to the conclusion about existence of some kind of a fractal transformation governing the energy transfer between the scales. This energy transfer may be considered analogous to the self-organized appearance of electromagnetic waves in resonator from background electromagnetic fluctuations in it. A wide spectrum of these fluctuations creates oscillations, whose frequency is determined by the spatial and construction parameters of the applied resonator.

This is, however, only an analogy. The energy transfer along the dynamic fractal spanned on several scales might be much more complicated than this. To describe the second approach of the section, one may discuss the disturbing side effects of this energy transfer towards microscales on some known experimental examples of so-called low-energy nuclear reactions (LENR), many of which are accompanied by the transmutation of involved substances. 
2.2. Self-Organized Dynamic Transfer Processes Possessing Fractal Geometries as Origin of LENR. Generally saying, the approach to the space in the theory [5] may be considered as an integral approach. Even if main description of a fractal character of the space refers to the concept of geodesics of elementary particles, a main part of the analysis in the theory [5] is devoted to the integral properties of such a space in application to the systems in relatively big scales. There is, however, definite evidence, that the fractal properties of space reveal itself not only in the mass distributions in astrophysical scales, considered in [5], but also in structuring in the quantum microscales, where geodesics of elementary particles form nuclei, atoms, and molecules. Because of uncertainty principle, one may freely assume that the geodesics of elementary particles span in the scales of engineering macrosystems. Due to this, the interaction of such macrosystems with presumably very fast motion of energy along the geodesics looks possible. Analysis of basics of the theory [5] provides with two main conditions for that

(1) fractal character of the geodesics,

(2) change of the fractal dimension of geodesics with change of the scales.

We would like to note that many dynamic processes on interfaces between gases, liquids, and solids provide with a fractal form of the processes. This form is also dynamically changing during the processes. This change may provide us with an accomplishment of the basic condition (2) leading to disturbing interaction with geodesics of some kind of elementary particles located in the spatial range of our engineering system, which contains fractals being dynamically created and changed.

By our opinion, there are several historically first examples of such engineering systems, which were described in the papers [10-12] with a misleading explanation of origin of LENR taking place in the systems. First of all, these systems are the systems with dynamically developing cracks in the solids doped by hydrogen or deuterium. Such a doping and detection of emission of neutrons and other elementary particles lead to search of possibilities for the hydrogen or deuterium fusion taking place with the help of a crystal lattice of the solids. By our opinion, this search is misleading. Main reason for this is well-known property of both gases to make the experimental solids brittle. Presence of atoms of these gases in a crystal lattice is presence of the defects, which promote development of fractures and cracks possessing a fractal geometry of their surfaces. An interaction between the fractal geodesics of elementary particles constituting substances of the solids and the fractures being dynamically developed in the solids provides with disturbing side effects on the structuring of the substances on a nuclear scale.

This interaction is also an alternative explanation for the transmutation phenomena observed in result of an explosive electrochemical destruction of metal foils in the experiments described in $[13,14]$. The main characteristics of the dynamically changing fractal interfaces in this case are not the interface between atoms of gas and solid, but the interface between atoms of gas and liquid, or between the electrons being moved by electromagnetic field and conductor, which form system with varying constraints.

The term constraints here is analogous to the same term in $[15,16]$, which generalizes results of experiments with relativistic electron beams focused on solid targets. Detailed outputs of the experiments are described in the book [17]. The interaction of a targeted substance with the high-energy focused electronic beam being constrained by the substance creates conditions for its transmutation and even for creations of super heavy nuclei, which are considered in [16] using the concepts of fractal isomers and clusters.

Despite the high energies involved, we may characterize these experiments as possessing the same general character of interaction between the competing agents forming mass transfer as in the case of generation of so-called fractal fingers, which are considered in the book [1] on the case of expunging of highly viscous liquid by the less viscous. The process is characterized as inherently nonequilibrium. It creates the dynamically changing fractal interface between two interacting substances. By our opinion, the dynamic properties of interactions between substances on two different sides of such an interface play the most important role in establishing interaction with the fractal geodesics.

An argument to support this conclusion may be found in the special dependencies of two closely connected phenomena taking place in the collapsing bubbles. The process of a bubble collapse may produce light [18] and neutrons [19]. In the first case the emission of photons from bubbles in water is highly dependent on presence of argon in the bubbles [20]. In the second case the dependence has been moved to another side of the interface-liquid. The latest experiments on generation of neutrons from the bubbles being created by powerful ultrasound [21] make evident critical dependency of this generation from the presence of salts of Fe in water. These experiments of the group researching the so-called piezonuclear reactions in the paper [21] support our conclusion about importance of the self-organized dynamics determined by the interacting substances residing on two sides of a dynamically changing fractal interface. This group recently has conducted experiments [22] using bar of steel subjected to a powerful ultrasound having the same frequency as applied to the salts of $\mathrm{Fe}$ in water. The same output as in [21] was detected in the form of bursts of neutrons. Authors of $[21,22]$ argue that neutrons are emitted in result of piezonuclear reactions, which are caused by extreme pressure and subsequent dislocations of adjacent atoms in the cases of imploding bubbles and violently deforming lattices of solids. Transmutation of substances in the steel also was detected in the latest experiments of the group [22]. The theory [23], which stays behind the experiments, considers the metrics of space time as dependent on energy values being involved into interactions. This is generally close to our own approach. Main difference, however, could be noted with regard to the engineering practice. The hypothesis of inherent fractal structure of space allows search of technically more feasible possibilities to alter its metrics. By this hypothesis the superhigh concentrations of energy in pretty small spatial areas are good, but not necessary for initiation of piezonuclear reactions. 


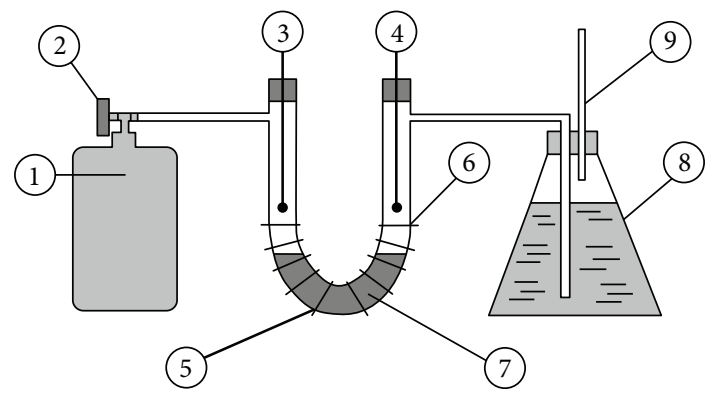

FIGURE 1: 1: volume containing propane/butane mixture; 2: valve to regulate flow of gas; $3,4,5$ : thermocouples; 6 : heating wire; 7 : $\mathrm{U}$ shape test glass with $\mathrm{Sn}$ : Bi alloy; 8: volume with water; 9: output tube.

By our opinion, the dynamic excitation of relatively big internal fractal structures of steel by ultrasound with subsequent development of fractal cracks accompanied by interaction between the atoms of $\mathrm{Fe}$ and atoms of some gas on the fractal interfaces might be responsible for the neutron bursts and transmutation. The same could be noted with regard to the fractal interfaces between gas and liquid in collapsing bubbles.

In addition to the considerations presented above, we would like to note that the discovered in $[21,22]$ side effects of the interactions between the fractal geodesics of constituents of experimental substances and their fractal interfaces may take place not only at the nuclear scale level. To find out such interactions, which create disturbing actions at some other scale levels of structuring of experimental substances, one may reproduce or conduct new experiments, which are close by their idea to the experiments described in the next section.

\section{Low-Temperature Pyrolysis of Hydrocarbons Using Dynamically Changing Fractal Interfaces}

Historically such pyrolysis, as designated in the title of this section, was conducted first by the owners of patent [24]. There was another theoretical explanation of the process. This explanation has put its main emphasis on the combinations of constituents of composite substances being in contact with the hydrocarbons, which were subjected to simultaneous action of dynamic potentials driving the heat and mass transfers. This theoretical explanation is presented on a personal site of one of coauthors of this paper (O.I. Vyhoniailo), who is also coowner of the patent [24]. Another explanation, which is presented in this paper, is illustrated by the following report about the concept of proof experiment. Next subsection after the report represents a short description of experimental predecessors for this experiment joined with a discussion.

3.1. A Concept Proof Experiment. The experimental setup for a concept proof experiment on low-temperature pyrolysis of gaseous hydrocarbons is presented in Figure 1.

The heating wire has been attached to the source of direct current. The number of heating wire windings around the test glass was tuned to support its constant temperature around

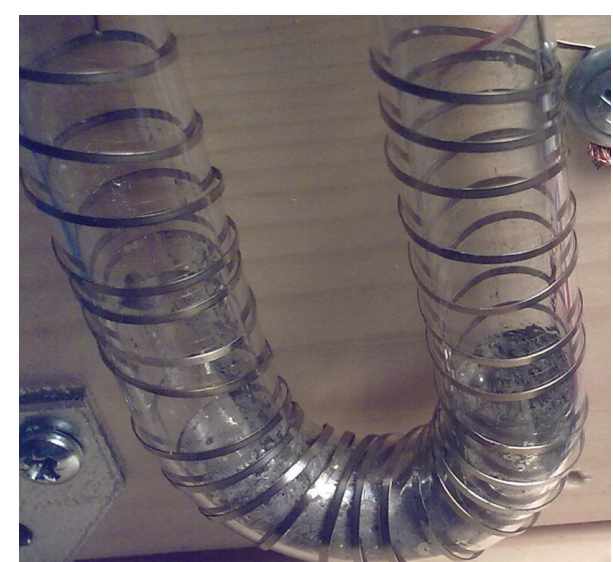

Figure 2: Carbon in the U tube.

the $200 \pm 20^{\circ} \mathrm{C}$ with the approximate flow of gas via the melted alloy $1 \mathrm{~mL} / \mathrm{sec}$. No thermocouple was submerged into the alloy. The setup was designed for a concept proof experiment. Therefore no gas composition measurements on output were made and estimations of the gas flow were performed using visual observations of the size and frequency of gas bubbles flowing from bottom of a volume with water to the open atmosphere via the output tube.

Such a simple experimental setup allows tuning of the gas flow via the alloy with direct observation of the carbon appearing on top of the metal from the right side of $U$ tube. Typical result of a short (10-15 minutes) experiment is shown by the photo in Figure 2. Here the alloy is cooled down. The temperatures measured by the three thermocouples were $186^{\circ} \mathrm{C}$ of gas coming to the alloy from the left input to the $\mathrm{U}$ tube, $204^{\circ} \mathrm{C}$ temperature of a heating wire, and $82^{\circ} \mathrm{C}$ of gas coming to the outlet at the right side of $\mathrm{U}$ tube. Short video of this experiment is presented in the Supplementary Material available online at http://dx.doi.org/10.1155/2013/310748.

The thermocouples were inserted to the $U$ tube via the gummy locks having drilled holes. As the thermocouples had two wires in a plastic insulation, they were inserted not totally tightly into the locks. Achievements of the stable temperature and gas flow regimes allowed the removal of the thermocouples and application of tightly set solid gummy locks. This allowed the detection of slow diffusion of gases remaining in the slang between the right side of $U$ tube and the volume with liquid. The diffusion was into the solidified alloy, which obtained high porosity with presumably fractal internal surfaces of the pores with carbon on their walls. One can see it in Figure 3 in the photo taken after 8 hours after the end of the carbon producing experiment. Water from the volume with it was sucked into the alloy.

We exclude significant effect on the sucking from a thermal decrease of a gas volume in the tubes due to the cooling, because we could not observe significant change of a water level in the volume on output during the time of complete cooling to the room temperature of all the parts of experimental setup.

Exact reason of this phenomenon is unknown, as detailed characterization of the alloy and its pores was not conducted. 


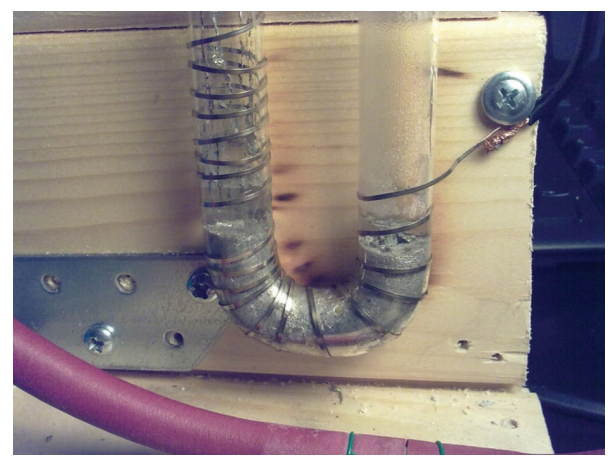

FIGURE 3: Water sucked by the alloy.

It was possible to observe using an optical microscope the highly developed fractal geometry of the pores with carbon on the metal after breaking of the glass with it. No analysis of internal porosity of the alloy was conducted. We make preliminary conclusion that main reason of sucking of water into the alloy might be in presence of a big internal volume in the fractal pores inside the alloy, which had mainly hydrogen and carbon during its solidification.

\subsection{Experimental History and Discussion. Historically the} same process (by the hypothesis) under the same temperatures took place in the experiments conducted prior filling of application for the patent [24]. The gas (methane) was moved by a mixer in the glass bulb along the surface of a mixture of fractured solids and porous salts, which were lying on the bottom of the bulb being heated by an electrical heater beneath the bulb. It is assumed from the common knowledge about fractals [1] that the substances lying on the bottom of the bulb possess the fractal geometry of their surface and pores. Temperature inside the bulb was monitored by a thermocouple inserted together with the mixer via lock at the top of the bulb. Carbon particles were formed directly in gas. Then they flew out of the bulb to the same volume with water as in the above-described experiment. With progress of methane pyrolysis the volume becomes dark being filled by the trapped particles. A short video of this experiment is presented in the supplementary material of this paper.

One may note that such an experiment has a difference with an alloy-based experiment in respect to the fractal interfaces. Here the interface is between the gas and solids, whose surfaces are assumed (not proved) to have a fractal geometry. The solids are also relatively stable. In contrast to this, the convective surface layers of gas being heated and moved in the bulb by a mixer with unavoidable turbulence definitely possess fractal properties of temperature and pressure fluctuations accordingly to many researches referenced in [25].

This note adds some ambiguity to the consideration regarding unstable fractal interfaces between the gas and liquid, as both phases may contribute to the dynamics on their interface. Yet it may provide another aspect to research of turbulence towards finding out of such possibilities, as described in the beginning of Section 2.
For example, if one will find out such a special regime of turbulence in the ionic flame-based magnetohydrodynamic generators, which obtains its support from accelerated reactions in lower scale of the fractal geodesics being disturbed, one may directly produce electrical energy from the energy of disturbed and decayed structures in lower scales. With regard to such a possibility one may develop an alternative explanation of acceleration of chemical reactions in reactive turbulent flows in comparison with the explanation based on the idea about an increase of mixing in turbulence.

Furthermore, if one will apply the approach to control the dynamic chaos of turbulence by the external perturbations analogous to the controlling actions of Nottale's hydraulic experiment [6], one may achieve intensification of the whole process and of the energy transfer process between the scales. By our opinion, such intensification takes place in the experiments with combustion of wood powder, which are described in [26]. The external perturbation, which stabilizes the flame, is applied to a burner in the form of low frequency sound (around $17 \mathrm{~Hz}$ ). It makes combustion much more intensive, and it increases the levels of NOx generation in the process. The large-scale dynamic parameters of the flame are changed drastically. Therefore it might be reasonable to conclude that analogous application of low-frequency sound in the same burner of a magnetohydrodynamic generator might bring unexpected increase of its efficiency.

Another prospective engineering system to explore the discussed side effects may be built using intentional creation of dynamic fractal structures in sound waves. This proposal logically follows from the notes about general ways of geometrisation of physics, discussed also by Nottale in his works. In context of these discussions, the geodesics may become the only entity, which is considered. In such a case the main meaning of the concept moves from the approach based on consideration of geodesics for particles in empty space to the approach based on consideration of purely relative differences between the geodesics, which are entangled in motion at several scale levels to form the space itself and to support their steady structures in the forms of particles and matter.

With respect to this particularity in the theoretical considerations, the self-organized fractal structures in turbulent flows and in convective surface layers become totally analogous to the fractal structures to be intentionally created in sound waves. Here the main role has to play the spatially oriented fractal structure of differences in pressure along some number of sound wave periods, many of which may have intentionally introduced disturbances of harmonic wave forms to create a dynamic fractal.

We do not know real engineering systems, which exactly utilize such an approach.

There is, however, one example of electromechanical system [27], which by opinion of its inventor and producers acts destructively on the hydrogen bonds in water. This destructive action is in possibility to produce atomic hydrogen, whose flows out of water purges various impurities from it. One may see various salts expunged from ordinary drink water on the photo in supplementary material of this paper. The photo of the device is presented along with another 
photo of volume with purified water having voltmeter in it. It shows 1.4 Volt of potential difference between the electrode in center of volume and metallic volume itself. Inventor of the device argued that this electric potential and cleaning of water appear due to the generation and motion of atomic hydrogen. As inventor was a medical doctor, the device has received its application as a physiotherapeutic device, which is certified for medical use in Kazakhstan. Nowadays this device is also applied for a fabrication of biologically active and purified bottled water.

The photo of volumes with water was taken from a report in popular Russian magazine about the technical exhibition, where the device was presented [28]. Photo of the device in a cabinet for physiotherapy was taken from one of the sites of its sellers [29].

By our opinion, the special action of the device on water is created by a passage of initially harmonic infrasound (around $14 \mathrm{~Hz}$ ) via specially designed composite substance. This composite substance modulates the infrasound in a special way due to its nonlinear mechanical properties. We may hypothesize about destructive effect on water from an excitation of internal fractal structures in the composite substance by the sound, and from modulation of this sound. Despite such an ambiguity, the effect on water being subjected to the sound, which is produced by the device [27], may be considered as analogous to the effect on hydrocarbons from the fractal structures in the convective layers between the gaseous hydrocarbons and $\mathrm{Sn}: \mathrm{Bi}$ alloy in our own experiments, or the sound waves resemble the form of macro quantum wave packets.

\section{Conclusions}

We have presented a general description of relatively rare engineering systems utilizing side effects arising from the dynamics involving self-organizing fractal interfaces. Both theoretical and practical backgrounds to explore and develop such systems were presented. We encourage readers to develop their own prospective engineering applications based on the presented theoretical approach and experimental examples, which were interpreted using this approach.

\section{Acknowledgments}

The authors would like to express their gratitude and thanks for a public discussion and experimental cooperation to another coowner of the patent [24], Shostak Taras Anatolievich, and to the Sintos Systems OU for financial support of this work.

\section{References}

[1] J. Feder, Fractals, Plenum Press, New York, NY, USA, 1991.

[2] A. Kurakin, "The self-organizing fractal theory as a universal discovery method: the phenomenon of life," Theoretical Biology and Medical Modelling, vol. 8, no. 1, article 4, 2011.

[3] C. T. Brown and W. R. T. Witschey, "The fractal geometry of ancient Maya settlement," Journal of Archaeological Science, vol. 30, no. 12, pp. 1619-1632, 2003.
[4] H. Haken, Synergetics, An Introduction: Nonequilibrium Phase Transitions and Self-Organization in Physics, Chemistry, and Biology, Springer, New York, NY, USA, 3rd edition, 1983.

[5] L. Nottale, "Scale relativity and fractal space-time: theory and applications," in Proceedings of 1st International Conference on the Evolution and Development of the Universe, vol. 15 of Foundation of Science, pp. 101-152, Paris, France, October 2008.

[6] L. Nottale and T. Lehner, "Numerical simulation of a macroquantum experiment: oscillating wave packet," International Journal of Modern Physics C, vol. 23, no. 5, Article ID 1250035, 27 pages, 2012.

[7] L. Notalle, "Generalized quantum potentials," Journal of Physics $A$, vol. 42, no. 27, pp. 275-306, 2009.

[8] A. Alevanau, W. Yang, W. Blasiak, and O. P. Kuznechik, "Prospective side effects of the heat and mass transfers in microporous structure of char during intermediate and final stages of the high-temperature pyrolysis," in Proceedings of the 16th International Conference School Foundations \& Advances in Nonlinear Science, Belarussian State University, Minsk, Belarus, 2012.

[9] R. Ball, A. C. McIntosh, and J. Brindley, "Feedback processes in cellulose thermal decomposition: implications for fire-retarding strategies and treatments," Combustion Theory and Modelling, vol. 8, no. 2, pp. 281-291, 2004.

[10] V. A. Klyuev, A. G. Lipson, P. Toporov et al., "High-energy processes accompanying the fracture of solids," Technical Physics Letters, vol. 12, pp. 551-552, 1986.

[11] B. V. Derjaguin, A. G. Lipson, V. A. Kluev, D. M. Sakov, and Y. P. Toporov, "Titanium fracture yields neutrons?" Nature, vol. 341, no. 6242 , p. $492,1989$.

[12] A. G. Lipson and G. H. Miley, "Phenomenon of an energetic charged particle emission from hydrogen/deuterium loaded metal," in Proceedings of the International Conference on Cold Fusion (ICCF '10), 2010.

[13] L. I. Urutskoev, V. I. Liksonov, and V. G. Tsinoev, "Observation of transformation of chemical elements during electric discharge," Annales de la Fondation Louis de Broglie, vol. 27, no. 4, pp. 701-726, 2002.

[14] L. I. Urutskoev, "Review of experimental results on low-energy transformation of nucleus," Annales de la Fondation Louis de Broglie, vol. 29, no. 3, pp. 1149-1164, 2004.

[15] S. Adamenko, V. Bolotov, and V. Novikov, "Basic principles of the concept of evolution of systems with varying constraints," in Control of Multiscale Systems with Constraints, V. Andruschenko, Ed., vol. 1 of Interdisciplinary Studies of Complex Systems, no. 1, pp. 33-54, Dragomanov National Pedagogical University, Kyiv, Ukraine, 2012.

[16] S. Adamenko, V. Bolotov, and V. Novikov, "Fractal nuclear isomers and clusters," in Control of Multiscale Systems with Constraints, V. Andruschenko, Ed., vol. 1 of Interdisciplinary Studies of Complex Systems, no. 1, pp. 55-77, Dragomanov National Pedagogical University, Kyiv, Ukraine, 2012.

[17] V. Adamenko, F. Selleri, and A. van der Merwe, Controlled Nucleosynthesis, Breakthroughs in Experiment and Theory, Springer, Dordrecht, The Netherlands, 2007.

[18] S. J. Putterman, "Sonoluminescence: sound into light," Scientific American, vol. 272, pp. 46-51, 1995.

[19] R. P. Taleyarkhan, C. D. West, J. S. Cho, R. T. Lahey, R. I. Nigmatulin, and R. C. Block, "Evidence for nuclear emissions during acoustic cavitation," Science, vol. 295, no. 5561, pp. 1868$1873,2002$. 
[20] D. Lohse, M. P. Brenner, T. F. Dupont, S. Hilgenfeldt, and B. Johnston, "Sonoluminescing air bubbles rectify argon," Physical Review Letters, vol. 78, no. 7, pp. 1359-1366, 1997.

[21] F. Cardone, G. Cherubini, R. Mignani et al., "Neutrons from piezonuclear reactions," Annales de la Fondation Louis de Broglie, vol. 34, no. 2, pp. 183-207, 2009.

[22] F. Cardone, R. Mignani, M. Monti, A. Petrucci, and V. Sala, "Piezonuclear neutrons from iron," Modern Physics Letters A, vol. 27, no. 18, Article ID 1250102, 11 pages, 2012.

[23] F. Cardone and R. Mignani, Energy and Geometry, World Scientific, 2004.

[24] O. I. Vyhoniailo and T. A. Shostak, "Process for the preparation of synthesis gas from fossil coal," UA79575, (C2), 2007, http:// v3.espacenet.com/origdoc? $\mathrm{DB}=\mathrm{EPODOC} \& \mathrm{IDX}=\mathrm{UA79575} \& \mathrm{~F}$ $=0 \& Q P N=U A 79575$.

[25] S. P. Malinowski and M. Y. Leclerc, "Fractal properties of temperature fluctuations in the convective surface layer," BoundaryLayer Meteorology, vol. 71, no. 1-2, pp. 169-187, 1994.

[26] B. Göktepe, R. Gebart, N. Leitão, I. V. Leitão, J. G. Merícia, and E. C. Fernandes, "Simultaneous pressure and heat release measurements in a $150 \mathrm{~kW}$ wood powder burner," in Proceedings of the SPEIC10: Towards Sustainable Combastion, Tenerife, Spain, June 2010.

[27] O. A. Kazakov, "Method for physiotherapy and device for its application," 003412 Kazkhstan, Eurasian, 2003.

[28] Board Editors, "Sensational discovery made by O. A. Kazakov in Kazakhstan," Tehnika Molodezhi, Vol. 2, 2002.

[29] Medpk, IFS-1, http://medpk.narod.ru/img_0407.jpg. 

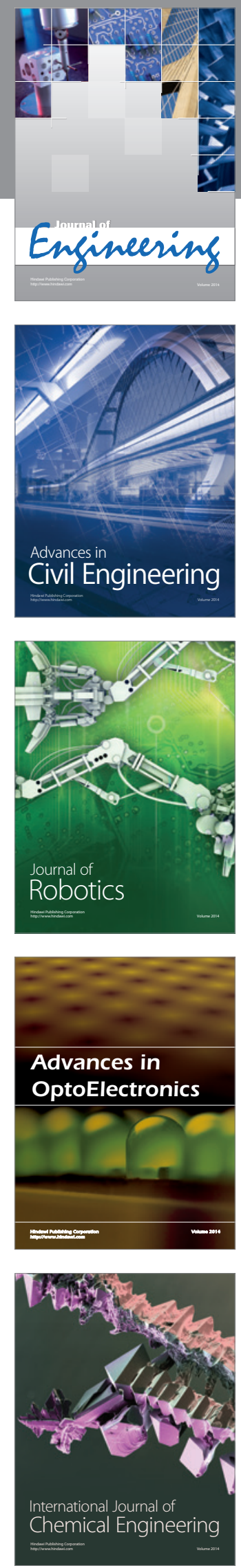

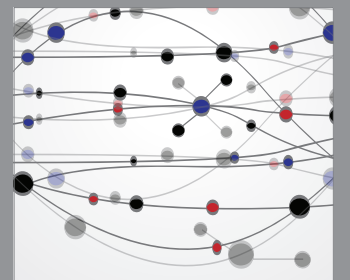

The Scientific World Journal
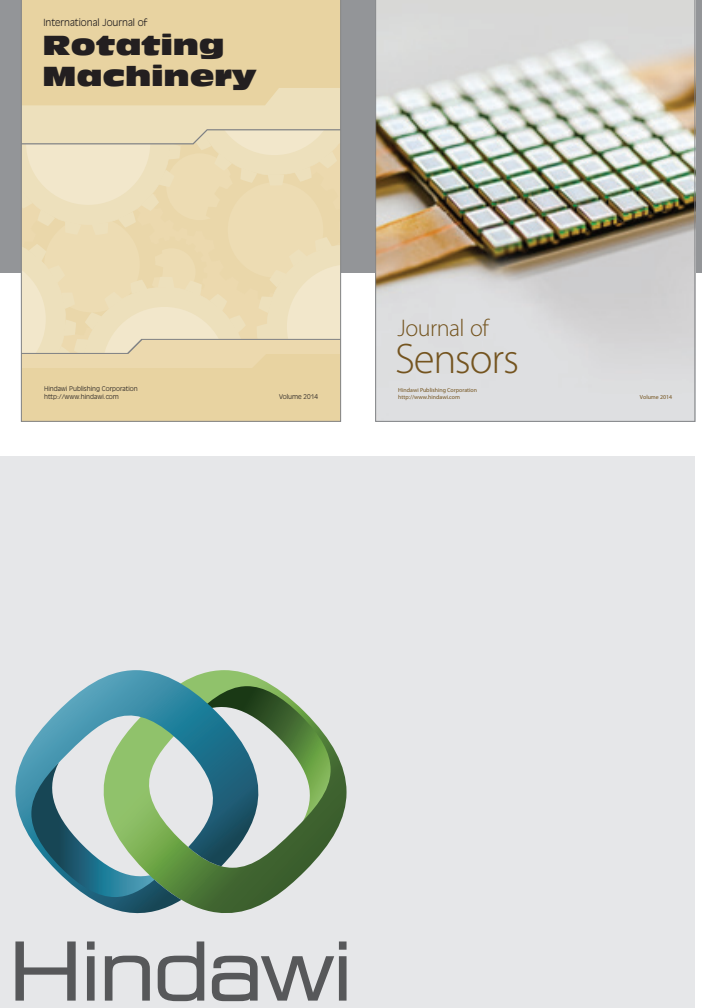

Submit your manuscripts at http://www.hindawi.com
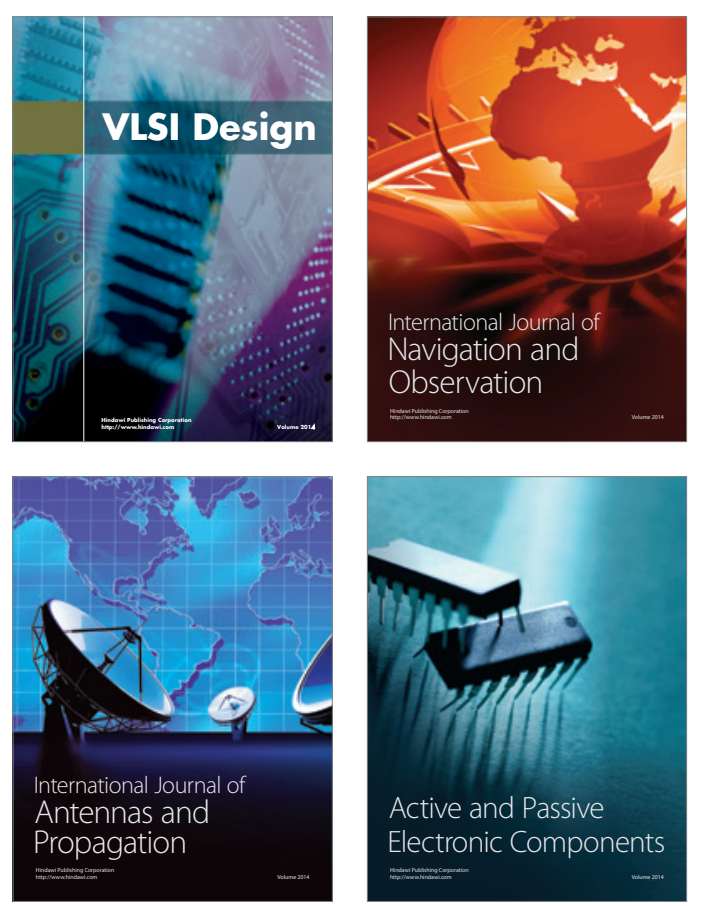
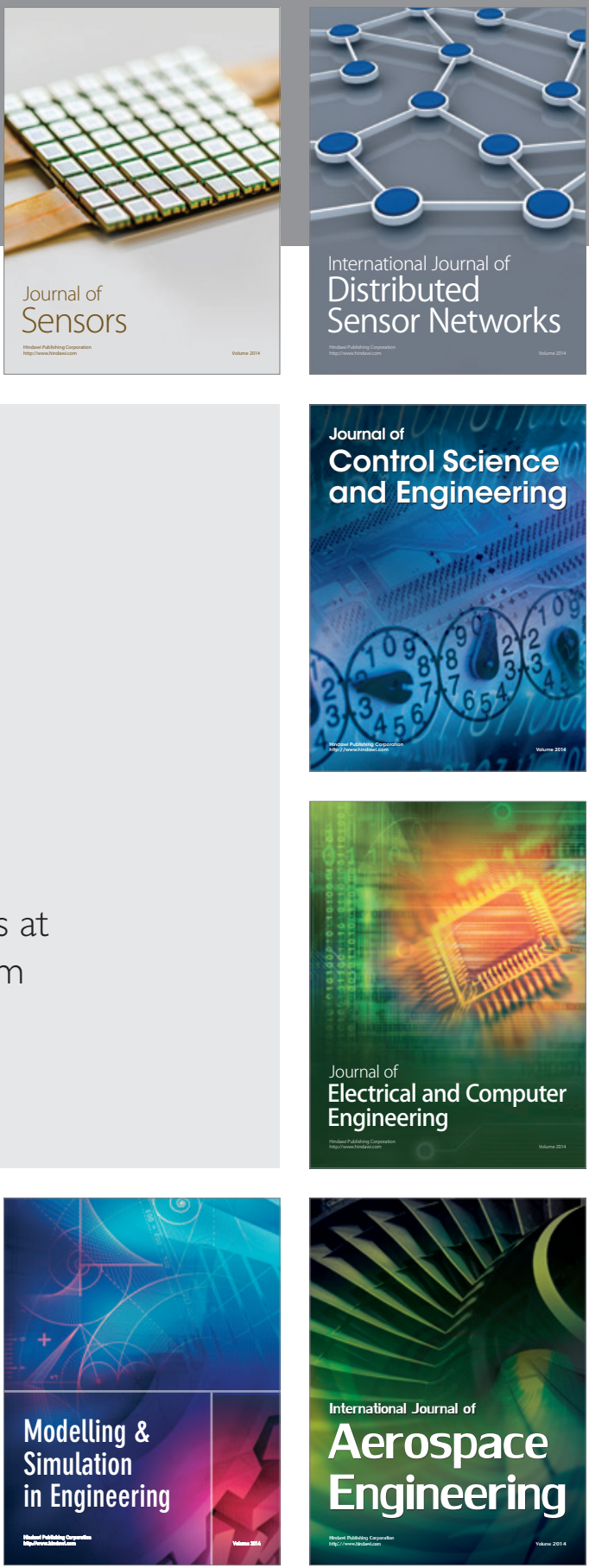

Journal of

Control Science

and Engineering
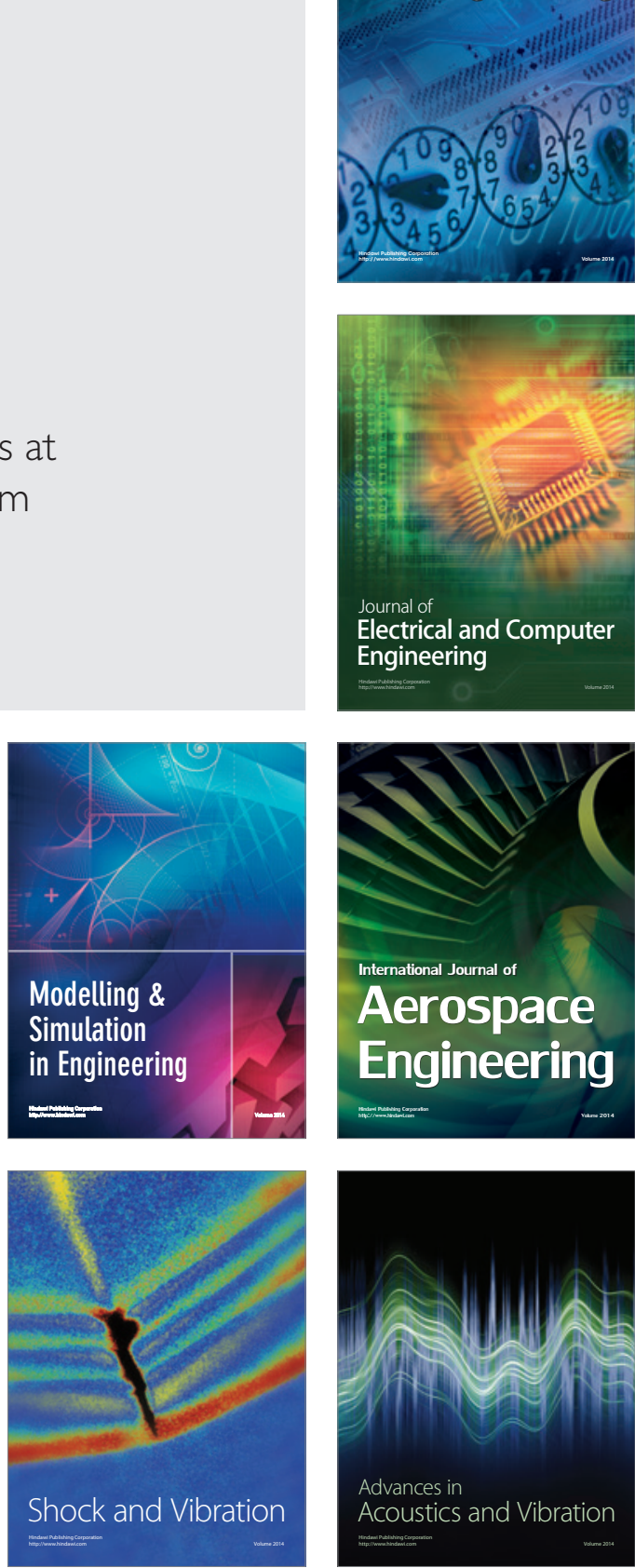